\title{
Editorial: Introduction to the 35th Annual Gallery of Fluid Motion (Denver, Colorado, USA, 2017)
}

\author{
Nicole S. Sharp ${ }^{1}$ and Patrick D. Weidman ${ }^{2}$ \\ ${ }^{1}$ FYFD, Denver, Colorado 80210, USA \\ ${ }^{2}$ University of Colorado Boulder, Boulder, Colorado 80309, USA
}

(Received 17 April 2018; published 17 October 2018)

DOI: 10.1103/PhysRevFluids.3.100001

The 35th Annual Gallery of Fluid Motion (GFM) was held at the American Physical Society (APS) Division of Fluid Dynamics (DFD) Annual Meeting in Denver, Colorado from November 19-21, 2017. The meeting featured approximately 2800 technical abstracts with an additional 106 entries to the GFM for display during the meeting (69 video and 37 poster submissions). Submissions were received from 23 countries with approximately $48 \%$ of submissions coming from institutions outside the United States. The works submitted represent a broad range of fluid dynamical topics being explored in academia, industry, and national laboratories today.

To reflect that diversity, the judging panels convened to select Gallery winners - 10 judges for the videos and 3 judges for the posters - included scholars from both academia and industry. In keeping with the GFM's purpose as a visual record of the aesthetic and science of contemporary fluid mechanics, judges were asked to evaluate submissions based on: (a) the beauty and aesthetic value of the work; (b) the scientific and technical merit of the described research; (c) originality and clarity in communication; (d) the approachability of the work to non-experts; and (e) the value in promoting the field of fluid dynamics to general audiences.

Videos were judged in two rounds. In the first round, each video was rated by three judges. In the second round, the top eleven videos were judged by all panelists. From these ratings, the top seven videos were chosen as winners. Posters were judged in person, with panelists selecting their rankings individually, then conferring on a final order to select four winning entries. As in previous years, the top three winners in each category were designated Milton van Dyke Award winners and received a monetary prize in addition to the certificates and copy of An Album of Fluid Motion given to all winners. The winning entries, as well as many of the other submissions, are visible online at http://gfm.aps.org. The 2017 Gallery of Fluid Motion winners are:

\section{Milton van Dyke Award video winners}

Marangoni bursting: Evaporation-induced emulsification of a two-component droplet. Guillaume Durey, Hoon Kwon, Quentin Magdelaine, Mathias Casiulis, Julien Mazet, Ludovic Keiser, Hadrien Bense, Pierre Colinet, José Bico, and Etienne Reyssat.

Symmetry breaking in Leidenfrost flows. Ambre Bouillant, Timothée Mouterde, Philippe Bourrianne, Christophe Clanet, and David Quéré.

Kicked drops. Pierre Chantelot, Martin Coux, Lucie Domino, Benoît Pype, Christophe Clanet, Antonin Eddi, and David Quéré. 


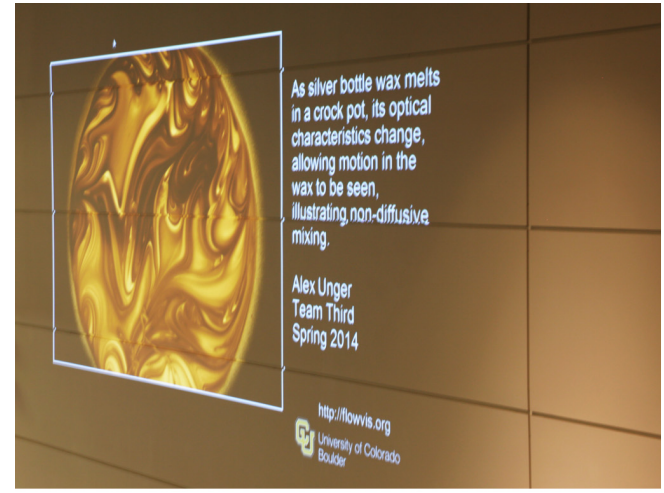

(a)

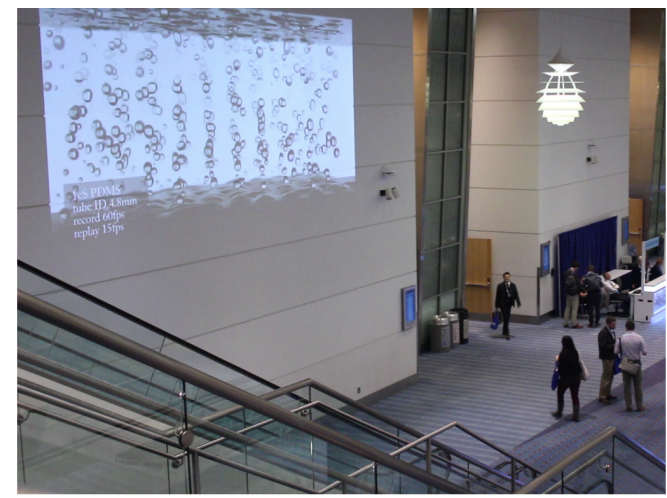

(b)

FIG. 1. Secondary displays in the publicly-accessible main concourse of the 2017 APS DFD Annual Meeting showing (a) student work from the Flow Visualization course as the University of Colorado, Boulder, and (b) video selections from previous years' Gallery of Fluid Motion submissions. Photo (a) taken by Larry E. Floyd. Photo (b) taken by Nicole Sharp.

\section{Milton van Dyke Award poster winners}

Fluted films. N. B. Speirs, M. M. Mansoor, J. Belden, R. C. Hurd, Z. Pan, and T. T. Truscott.

Mandala-inspired representation of the turbulent energy cascade. Maxime Bassenne, Hyunji Jane Bae, and Adrián Lozano-Durán.

Solid structures generated by capillary instability in thin liquid films. J. Marthelot, E. F. Strong, P. M. Reis, and P.-T. Brun.

\section{Gallery of Fluid Motion Award video winners}

Soap opera in the maze: Geometry matters in Marangoniflows. Fernando Temprano-Coleto, François J. Peaudecerf, Julien R. Landel, Frédéric Gibou, and Paolo Luzzatto-Fegiz.

Spin lattices of walking droplets. Pedro J. Sáenz, Giuseppe Pucci, Alexis Goujon, Tudor CristeaPlaton, Jörn Dunkel, and John W. M. Bush.

Emergence of small scales in vortex ring collisions. Ryan McKeown, Rodolfo Ostilla-Mónico, Alain Pumir, Michael P. Brenner, and Shmuel M. Rubinstein.

Coexistence of order and chaos in C major. Paul M. Branson, M. Rayson, M. Ghisalberti, and G. N. Ivey.

\section{Gallery of Fluid Motion Award poster winners}

Splash of milk streams impacting on a solid surface. Azar Eslam-Panah and Heidi E. Reuter.

In addition to the main display of this year's GFM submissions, the Annual Meeting contained two secondary displays in the main concourse, shown in Fig. 1. One display, curated by Jean Hertzberg, featured a collection of student work from the Flow Visualization course at the University of Colorado, Boulder. ${ }^{1}$ The second display, curated by Nicole Sharp, showed 50 videos from 11 years' worth of previous GFM submissions. Funding for the displays was generously provided by the University of Colorado, Boulder.

\footnotetext{
${ }^{1}$ See http://www.flowvis.org to view the full collection.
} 
To conclude, we extend our sincere thanks to all the contributors to the 2017 GFM and to our thirteen judges. Special thanks are due to Kenneth Kiger, whose continued service as overall coordinator for the GFM ensures institutional memory and helps guide each local organizing committee in bringing to life a new but consistent iteration of the Gallery. We are grateful to the meeting co-Chairs, Jean Hertzberg and Peter Hamlington, for their trust and support. We would also like to thank Paul Dlug and Mark Doyle of APS for their continuing management of the GFM website, Peggy Holland and Margaret McDonald of Meetings and More for their logistical mastery of all details both large and small, and the staff of APS for their dedicated assistance and coordination throughout the GFM cycle. 